\title{
Permanent magnet synchronous machines flux linkage estimation with zero steady-state error and its field-programmable gate array implementation
}

ISSN $1751-8660$

Received on 5th February 2014 Accepted on 1st December 2014 doi: 10.1049/iet-epa.2014.0241 www.ietdl.org

\author{
Giovanni Zanuso ${ }^{1}$, Luca Peretti ${ }^{1}$, Mauro Zigliotto ${ }^{2}$ \\ ${ }^{1}$ Department of Electrical Systems, ABB Corporate Research, Forskargränd 7, 72178 Västerås, Sweden \\ ${ }^{2}$ Department of Technique and Management of Industrial Systems, University of Padova, Stradella San Nicola 3, 36100 Vicenza, Italy \\ 凶-mail: luca.peretti@se.abb.com
}

\begin{abstract}
The paper analyses a combined current model-voltage model estimator for flux linkages in permanent magnet synchronous machines, with the capability of converging to exact flux estimation even in presence of mismatches because of magnetic saturation. As a trend in next-generation electric drives, the whole algorithm, including both the flux estimator and the standard field-oriented control, has been implemented in a field-programmable gate array (FPGA) chip. Simulations and experimental tests, along with some figures for the FPGA selection, have been included in the study.
\end{abstract}

\section{Introduction}

The correct estimation of flux linkages in permanent magnet synchronous machines is a technical enabler for a variety of improvements in modern $\mathrm{AC}$ drives, including torque estimation, efficient control, faster dynamics and sensorless control.

As trivial as it might look, the flux linkage estimation has to cope with many technical issues and it still arises the research interest [1, 2]. A common one is the saturation of the magnetic circuit within the machine as function of the operating point, which could also result in cross-magnetisation effects because of the mutual coupling between the $d$ and $q$ axes of the machine. Such effects are well described in past literature works, as for example in [3-7]. The presence of saturation makes the estimation of fluxes based on measured currents slightly more complicated than a simple linear relationship $\lambda=L i$, where $\lambda$ is a generic flux, $L$ is an inductance and $i$ represents the measured current.

Moreover, synchronous machines with magnets may change the value of the magnet flux linkage because of temperature variations and ageing [8-10]. This effect has to be taken into account considering the application of electrical drives in demanding environments, such as traction applications [11].

Errors in the current model might be partially compensated by the exploitation of a flux linkage estimation based on the integration of the back electromotive force (bemf), which is a well-known approach. The main drawback of these models resides in the knowledge of the phase voltage, which must be cleaned from the spurious effects of inverter dead time distortion and PWM delays [12]. Another problem is the stator resistance variation with temperature, which brings to inaccurate estimations especially in the low-speed region [13].

Considering the above mentioned estimation approaches, and their related issues, different techniques are applied for the on-line parameter estimation of flux linkages: model-reference adaptive systems [14], Kalman filters [14], artificial neural networks [15], recursive least square error methods [16], signal injection methods [17], observers based on Lyapunov theory [18], estimators based on machine equations [19-21]. Some approaches consider constant inductances, as [13, 22], limiting their utilisation to systems on which there exists some prior knowledge of the machine characteristics. Other solutions, for example such as [19], combine the machine equations to very specific features, such as the amplitude of the main harmonics in the bemf. Others, such as $[20,21]$, rely on the knowledge of the stator resistance and try to perform a flux linkage estimation even in saturating conditions. In particular, [20, 21] perform an estimation by combining a current model for the low-speed region and a voltage model for the high-speed region, discriminating the output by means of a second-order filter. Such approach has been further investigated in [23], trying to overcome some small discrepancies occurred in the original works, to obtain an error-free estimation at steady state.

This paper gives further insights on [23], by enlarging its theoretical foundations and comparison with [20, 21], finding a solution to the steady-state errors that potentially affected those solutions. A detailed theoretical and experimental sensitivity analysis is included. As a recent trend for the next-generation $\mathrm{AC}$ drives, the whole algorithm, including the conventional field oriented control in which the estimator is merged, has been implemented on a field-programmable gate array (FPGA) chip. The reasons for the migration from standard microcontrollers to all-on-chip FPGA implementation are manifold. Among them, the fast execution time, the inherent protection of intellectual property (IP), improved software portability, possible mass-production savings, almost device-independent source code. Among cons, a certain difference in software structure with respect to microprocessor sequential codes, especially as regards to the timing diagram of the functional blocks and still a general complexity in estimating in advance the potentiality of the resources of a given FPGA chip. In view of an industrial exploitation of the estimator, the aforementioned items pushed towards a tentative FPGA implementation. It produced some useful design hints about the timing diagram and the needed resources, both included in the present work.

The paper is organised as follows. The theory is briefly recalled in Section 2. Section 3 is devoted to the theoretical parameter sensitivity analysis. A comparison between the original and the proposed solution is performed with simulations in Section 4. The laboratory test bench and the FPGA implementation are discussed in Section 5, which contains also the experimental tests and the experimental sensitivity analysis. Some conclusive remarks end the paper. 


\section{Theory}

\subsection{Electrical machine equations}

The equation describing the time-domain dynamics of a synchronous machine in matrix notation and in a stator-fixed $\alpha \beta$ reference frame is as follows

$$
\boldsymbol{u}_{\alpha \beta}=R_{s} \boldsymbol{i}_{\alpha \beta}+\frac{\mathrm{d} \boldsymbol{\lambda}_{\alpha \beta}}{\mathrm{d} t}
$$

where $R_{s}$ is the stator resistance, whereas $\boldsymbol{u}_{\alpha \beta}=\left[u_{\alpha} u_{\beta}\right]^{\mathrm{T}}$,

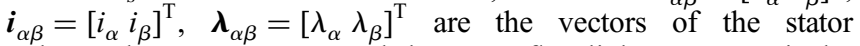
voltage, the stator current and the stator flux linkage, respectively. A more convenient description in the $d q$ reference frame, rotating with the electro-mechanical rotor angle $\vartheta_{\text {me }}$, is given

$$
\boldsymbol{u}_{d q}=R_{s} \boldsymbol{i}_{d q}+\frac{\mathrm{d} \boldsymbol{\lambda}_{d q}}{\mathrm{~d} t}+\omega_{\mathrm{me}} \boldsymbol{J} \boldsymbol{\lambda}_{d q}
$$

where $\omega_{\text {me }}$ is the electro-mechanical rotor speed, $\boldsymbol{J}=[0-1 ; 10]$ is the matrix responsible for the cross-coupling effect, whereas $\boldsymbol{u}_{d q}=\left[\begin{array}{ll}u_{d} & u_{q}\end{array}\right]^{\mathrm{T}}, \boldsymbol{i}_{d q}=\left[\begin{array}{ll}i_{d} & i_{q}\end{array}\right]^{\mathrm{T}}, \boldsymbol{\lambda}_{d q}=\left[\begin{array}{ll}\lambda_{d} & \lambda_{q}\end{array}\right]^{\mathrm{T}}$ are the $d q$ vectors of the stator voltages, the stator currents and the stator flux linkages, respectively. The components of $\boldsymbol{\lambda}_{d q}$, namely $\lambda_{d}$ and $\lambda_{q}$, are non-linear functions of the components of $\boldsymbol{i}_{d q}$, namely $i_{d}$ and $i_{q}$, because of the magnetic saturation

$$
\begin{aligned}
& \lambda_{d}=f_{d}\left(i_{d}, i_{q}\right)+\lambda_{\mathrm{mg}}\left(i_{d}, i_{q}\right) \\
& \lambda_{q}=f_{q}\left(i_{d}, i_{q}\right)
\end{aligned}
$$

where $\lambda_{\mathrm{mg}}$ is the permanent magnet flux linkage. If a linear magnetic model is used, $f_{d}\left(i_{d}, i_{q}\right)=L_{d} i_{d}, f_{q}\left(i_{d}, i_{q}\right)=L_{q} i_{q}$ and $\lambda_{\mathrm{mg}}\left(i_{d}, i_{q}\right)=\lambda_{\mathrm{mg}}$, where $L_{d}$ and $L_{q}$ are the inductances on the $d$ and $q$ axis, respectively. The system description is completed with the torque and the mechanical time-domain equations

$$
\begin{aligned}
\tau_{m} & =\frac{3}{2} p\left(\lambda_{d} i_{q}-\lambda_{q} i_{d}\right) \\
\tau_{m} & =J_{m} \frac{\mathrm{d} \omega_{m}}{\mathrm{~d} t}+B_{m} \omega_{m}+\tau_{L}
\end{aligned}
$$

where $\tau_{m}$ is the machine torque, $p$ is the pole pairs number, $J_{m}$ is the mechanical inertia, $B_{m}$ is the mechanical viscous friction and $\omega_{m}$ is the mechanical speed equal to $\omega_{\mathrm{me}} / p$.

\subsection{Flux linkage estimation}

An on-line flux linkage estimation can be based on (1), where $\boldsymbol{i}_{\alpha \beta}$ is measured and the voltage $\boldsymbol{u}_{\alpha \beta}$ is equal to the voltage reference generated by a conventional current regulator, if dead times and delays introduced by the inverter are compensated. Thus, in the Laplace-transform domain, it follows

$$
\hat{\boldsymbol{\lambda}}_{\alpha \beta, v}=\frac{1}{S}\left(\boldsymbol{u}_{\alpha \beta}-\hat{R}_{s} \boldsymbol{i}_{\alpha \beta}\right)
$$

where $\hat{\boldsymbol{\lambda}}_{\alpha \beta, v}=\left[\hat{\lambda}_{\alpha, v} \hat{\lambda}_{\beta, v}\right]^{\mathrm{T}}$ is the estimated flux from the so-called voltage model (5), with $\hat{R}_{s}$ as the estimated stator resistance. The integration in (5) makes the voltage model sensitive to possible mismatches in the stator resistance estimation and the unavoidable presence of spurious DC components in the current measurements. Inaccuracies might be larger at lower speeds, where the voltages are smaller and potential DC errors or resistance mismatches are heavier. Of course, the discrete-time implementation may cause additional problems.

The flux linkage can be also estimated from (3), assuming magnetic linearity and the knowledge of the inductances and of the magnets flux

$$
\hat{\boldsymbol{\lambda}}_{d q, c}=\hat{\boldsymbol{L}}_{d q} \boldsymbol{i}_{d q}+\hat{\boldsymbol{\lambda}}_{\mathrm{mg}}=\left[\begin{array}{cc}
\hat{L}_{d} & 0 \\
0 & \hat{L}_{q}
\end{array}\right]\left[\begin{array}{c}
i_{d} \\
i_{q}
\end{array}\right]+\left[\begin{array}{c}
\hat{\boldsymbol{\lambda}}_{\mathrm{mg}} \\
0
\end{array}\right]
$$

where $\hat{\boldsymbol{\lambda}}_{d q, c}$ is the estimated flux from the so-called current model (6), $\hat{L}_{d}$ and $\hat{L}_{q}$ are the estimated inductances on $d$ and $q$ axis, respectively, and $\hat{\lambda}_{\mathrm{mg}}$ is the estimated value of the permanent magnet flux linkage. The presence of constant inductances $\hat{L}_{d}$ and $\hat{L}_{q}$ makes the current model inaccurate in presence of magnetic saturation. Despite this, at low speeds the current model (6) might be preferable to the voltage model (5), because no integral operation is required.

In [20,21], the voltage and current models are initially merged by using a high-pass filter for the voltage model and a low-pass filter for the current model for a more robust flux estimation. In the Laplace transform domain, this is expressed by

$$
\begin{aligned}
\hat{\boldsymbol{\lambda}}_{\alpha \beta}= & {\left[\begin{array}{cc}
\frac{s^{2}}{s^{2}+2 \xi \omega_{0} s+\omega_{0}^{2}} & 0 \\
0 & \frac{s^{2}}{s^{2}+2 \xi \omega_{0} s+\omega_{0}^{2}}
\end{array}\right] \hat{\boldsymbol{\lambda}}_{\alpha \beta, v} } \\
& +\left[\begin{array}{cc}
\frac{2 \xi \omega_{0} s+\omega_{0}^{2}}{s^{2}+2 \xi \omega_{0} s+\omega_{0}^{2}} & 0 \\
0 & \frac{2 \xi \omega_{0} s+\omega_{0}^{2}}{s^{2}+2 \xi \omega_{0} s+\omega_{0}^{2}}
\end{array}\right] \hat{\boldsymbol{\lambda}}_{\alpha \beta, c} \\
= & {\left[\begin{array}{cc}
G_{\alpha \beta, v} & 0 \\
0 & G_{\alpha \beta, v}
\end{array}\right] \hat{\boldsymbol{\lambda}}_{\alpha \beta, v}+\left[\begin{array}{cc}
G_{\alpha \beta, c} & 0 \\
0 & G_{\alpha \beta, c}
\end{array}\right] \hat{\boldsymbol{\lambda}}_{\alpha \beta, c} } \\
= & \boldsymbol{G}_{\alpha \beta, v} \hat{\boldsymbol{\lambda}}_{\alpha \beta, v}+\boldsymbol{G}_{\alpha \beta, c} \hat{\boldsymbol{\lambda}}_{\alpha \beta, c}
\end{aligned}
$$

where $\hat{\boldsymbol{\lambda}}_{\alpha \beta}=\left[\hat{\lambda}_{\alpha} \hat{\lambda}_{\beta}\right]^{\mathrm{T}}$ and $\hat{\boldsymbol{\lambda}}_{\alpha \beta, c}=\left[\hat{\lambda}_{\alpha, c} \hat{\lambda}_{\beta, c}\right]^{\mathrm{T}}$ are the estimated flux and the current model flux in the $\alpha \beta$ reference frame, whereas $\xi$ and $\omega_{0}$ are, respectively, the damping ratio and the crossover frequency of the filters. The time-domain representation of (7) is

$$
\begin{aligned}
\hat{\boldsymbol{\lambda}}_{\alpha \beta}(t)= & \boldsymbol{g}_{\alpha \beta, v}(t) * \hat{\boldsymbol{\lambda}}_{\alpha \beta, v}(t)+\boldsymbol{g}_{\alpha \beta, c}(t) * \hat{\boldsymbol{\lambda}}_{\alpha \beta, c}(t) \\
= & \int_{-\infty}^{+\infty} \boldsymbol{g}_{\alpha \beta, v}(t-\tau) \hat{\boldsymbol{\lambda}}_{\alpha \beta, v}(\tau) \mathrm{d} \tau \\
& +\int_{-\infty}^{+\infty} \boldsymbol{g}_{\alpha \beta, c}(t-\tau) \hat{\boldsymbol{\lambda}}_{\alpha \beta, c}(\tau) \mathrm{d} \tau
\end{aligned}
$$

where $*$ is the convolution operator, and $\boldsymbol{g}_{\alpha \beta, v}$ and $\boldsymbol{g}_{\alpha \beta, c}$ are the time-domain representations of $\boldsymbol{G}_{\alpha \beta, v}$ and $\boldsymbol{G}_{\alpha \beta, c}$, respectively. The $d q$ reference frame representation is obtained as

$$
\begin{aligned}
\hat{\boldsymbol{\lambda}}_{d q}(t)= & \boldsymbol{T}_{\alpha \beta / d q}(t)\left[\int_{-\infty}^{+\infty} \boldsymbol{g}_{\alpha \beta, v}(t-\tau) \boldsymbol{T}_{d q / \alpha \beta}(\tau) \hat{\boldsymbol{\lambda}}_{d q, v}(\tau) \mathrm{d} \tau\right. \\
& \left.+\int_{-\infty}^{+\infty} \boldsymbol{g}_{\alpha \beta, c}(t-\tau) \boldsymbol{T}_{d q / \alpha \beta}(\tau) \hat{\boldsymbol{\lambda}}_{d q, c}(\tau) \mathrm{d} \tau\right]
\end{aligned}
$$

where $\boldsymbol{T}_{d q / \alpha \beta}$ and $\boldsymbol{T}_{\alpha \beta / d q}$ are the transformation matrices from $\alpha \beta$ to $d q$ and from $d q$ to $\alpha \beta$, respectively. By considering that $\boldsymbol{T}_{\alpha \beta / d q}(t) \boldsymbol{T}_{d q / \alpha \beta}(\tau)=$ $\boldsymbol{T}_{\alpha \beta / d q}(t-\tau)$ and that $\boldsymbol{g}_{\alpha \beta, v}, \boldsymbol{g}_{\alpha \beta, c}$ are scalar matrices, it follows

$$
\begin{aligned}
\hat{\boldsymbol{\lambda}}_{d q}(t)= & \int_{-\infty}^{+\infty} \boldsymbol{T}_{\alpha \beta / d q}(t-\tau) \boldsymbol{g}_{\alpha \beta, v}(t-\tau) \hat{\boldsymbol{\lambda}}_{d q, v}(\tau) \mathrm{d} \tau \\
& +\int_{-\infty}^{+\infty} \boldsymbol{T}_{\alpha \beta / d q}(t-\tau) \boldsymbol{g}_{\alpha \beta, c}(t-\tau) \hat{\boldsymbol{\lambda}}_{d q, c}(\tau) \mathrm{d} \tau
\end{aligned}
$$

Thus, in the Laplace transform domain, the expression (10) becomes

$$
\hat{\boldsymbol{\lambda}}_{d q}=\boldsymbol{G}_{d q, v} \hat{\boldsymbol{\lambda}}_{d q, v}+\boldsymbol{G}_{d q, c} \hat{\boldsymbol{\lambda}}_{d q, c}
$$


where $\boldsymbol{G}_{d q, v}=\mathcal{L}\left[\boldsymbol{T}_{\alpha \beta / d q}(t) \boldsymbol{g}_{\alpha \beta, v}(t)\right]$ and $\boldsymbol{G}_{d q, c}=\mathcal{L}\left[\boldsymbol{T}_{\alpha \beta / d q}(t) \boldsymbol{g}_{\alpha \beta, c}(t)\right]$, with $\mathcal{L}[f(t)]$ being the Laplace transform of the generic function $f(t)$. The transform $\boldsymbol{T}_{d q / \alpha \beta}(t)$ could be rewritten by considering that any multiplication with sine and cosine functions is a multiplication with exponential functions according to the Euler formulae

$\cos \left(\omega_{\mathrm{me}} t\right)=\frac{\mathrm{e}^{\mathrm{j} \omega_{\mathrm{me}} t}+\mathrm{e}^{-\mathrm{j} \omega_{\mathrm{me}} t}}{2}, \quad \sin \left(\omega_{\mathrm{me}} t\right)=\frac{\mathrm{e}^{\mathrm{j} \omega_{\mathrm{me}} t}-\mathrm{e}^{-\mathrm{j} \omega_{\mathrm{me}} t}}{2 j}$

By exploiting to the following Laplace transform property

$$
\int_{0}^{+\infty} f(t) \mathrm{e}^{\mathrm{at}} \mathrm{e}^{-\mathrm{st}} \mathrm{d} t=\mathcal{L}[f(t)](s-a)
$$

the expressions for $\boldsymbol{G}_{d q, v}$ and $\boldsymbol{G}_{d q, c}$ in (11) are found

$$
\begin{aligned}
& \boldsymbol{G}_{d q, v}=\frac{1}{2}\left[\begin{array}{ll}
{\left[G_{\alpha \beta, v}\left(s^{-}\right)+G_{\alpha \beta, v}\left(s^{+}\right)\right]} & -j\left[G_{\alpha \beta, v}\left(s^{-}\right)-G_{\alpha \beta, v}\left(s^{+}\right)\right] \\
j\left[G_{\alpha \beta, v}\left(s^{-}\right)-G_{\alpha \beta, v}\left(s^{+}\right)\right] & {\left[G_{\alpha \beta, v}\left(s^{-}\right)+G_{\alpha \beta, v}\left(s^{+}\right)\right]}
\end{array}\right] \\
& \boldsymbol{G}_{d q, c}=\frac{1}{2}\left[\begin{array}{ll}
{\left[G_{\alpha \beta, c}\left(s^{-}\right)+G_{\alpha \beta, c}\left(s^{+}\right)\right]} & -j\left[G_{\alpha \beta, c}\left(s^{-}\right)-G_{\alpha \beta, c}\left(s^{+}\right)\right] \\
j\left[G_{\alpha \beta, c}\left(s^{-}\right)-G_{\alpha \beta, c}\left(s^{+}\right)\right] & {\left[G_{\alpha \beta, c}\left(s^{-}\right)+G_{\alpha \beta, c}\left(s^{+}\right)\right]}
\end{array}\right]
\end{aligned}
$$

where, for ease of notation, it was set $\left(s-j \omega_{\mathrm{me}}\right)=s^{-}$and $\left(s+j \omega_{\mathrm{me}}\right)=s^{+}$.

The estimated flux in (7) and (11) is still incorrect if the estimated parameters $\hat{L}_{d}, \hat{L}_{q}, \hat{\lambda}_{\mathrm{mg}}$ and $\hat{R}_{s}$ are not accurate. In [21], the inaccuracies (excluding the $\hat{R}_{s}$ case) are compensated by considering that a current regulation in conventional FOCs, at steady-state, will generate a voltage reference vector equal to the right term of (2) without the derivative term. If the parameters are accurate, and a decoupling strategy of the $d q$ axes is applied, the output of the current PI regulators is equal to the resistive drop only. On the other hand, any parameter inaccuracy is compensated by the current regulation and reflected in the PI regulator output, which contains both the resistive drop and an additional term $\omega_{\text {me }} \boldsymbol{J} \Delta \boldsymbol{\lambda}_{d q}$, with $\Delta \boldsymbol{\lambda}_{d q}=\boldsymbol{\lambda}_{d q}-\hat{\boldsymbol{\lambda}}_{d q}$ where $\hat{\boldsymbol{\lambda}}_{d q}=\left[\hat{\boldsymbol{\lambda}}_{d} \hat{\boldsymbol{\lambda}}_{q}\right]^{\mathrm{T}}$ is the estimated flux in $d q$. It is therefore possible to obtain a flux compensating term from the output of the PI regulator, according to

$$
\Delta \boldsymbol{\lambda}_{d q}=\frac{1}{\omega_{\mathrm{me}}} \boldsymbol{J}^{-1}\left(\boldsymbol{u}_{d q, \text { int }}-\hat{R}_{s} \boldsymbol{i}_{d q}\right)=-\frac{1}{\omega_{\mathrm{me}}} \boldsymbol{J}\left(\boldsymbol{u}_{d q, \text { int }}-\hat{R}_{s} \boldsymbol{i}_{d q}\right)
$$

Actually, only the output of the integral part $\boldsymbol{u}_{d q \text {,int }}=\left[u_{d \text {,int }} u_{q \text {,int }}\right]^{\mathrm{T}}$ is used, whereas the proportional part (null at steady state) is usually omitted, because of its noisy behaviour (see [21] for more details). In [21], the term (15) is added to the output of the current model (6), modifying (11) as follows

$$
\hat{\boldsymbol{\lambda}}_{d q}=\boldsymbol{G}_{d q, v} \hat{\boldsymbol{\lambda}}_{d q, v}+\boldsymbol{G}_{d q, c} \hat{\boldsymbol{\lambda}}_{d q, c}+\boldsymbol{G}_{d q, c} \Delta \boldsymbol{\lambda}_{d q}
$$

Section 3 clarifies that the flux estimation in (16) does not assure perfect convergence to the actual flux linkage in presence of parameter mismatch. Therefore a modification is proposed and analysed in detail in Section 2.3.

\subsection{Proposed flux linkage estimation}

The flux estimator described in (16) is modified by processing the additional term $\Delta \boldsymbol{\lambda}_{d q}$ with a PI regulator and by adding it at the output of both the voltage and current models in $d q$. Therefore the proposed flux linkage estimation modifies the (16) according to the following expression

$$
\hat{\boldsymbol{\lambda}}_{d q}=\boldsymbol{G}_{d q, v} \hat{\boldsymbol{\lambda}}_{d q, v}+\boldsymbol{G}_{d q, c} \hat{\boldsymbol{\lambda}}_{d q, c}+\left(K_{p}+\frac{K_{i}}{s}\right) \Delta \boldsymbol{\lambda}_{d q}
$$

A convenient implementation of the proposed flux estimator, which will be used in the sensitivity analysis of Section 3, is shown in Fig. 1.

\section{Parameter sensitivity analysis}

\subsection{Analysis of $\boldsymbol{G}_{d q, \mathrm{c}}$ and $\boldsymbol{G}_{d q, v}$}

Understanding the proposed flux estimator requires a preliminary analysis of $\boldsymbol{G}_{d q, c}$ and $\boldsymbol{G}_{d q, v}$ in (14) and (17). The (14) can be

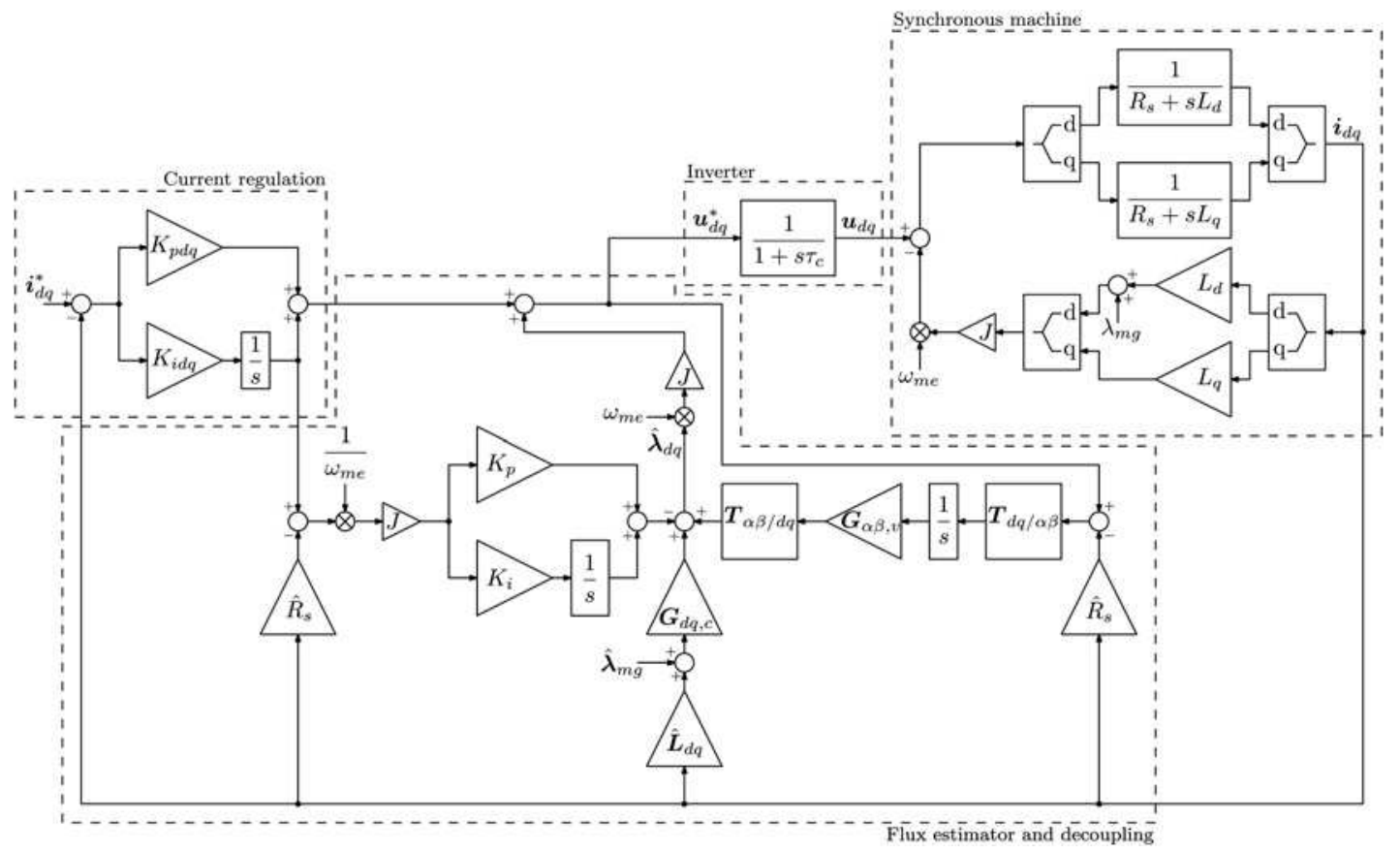

Fig. 1 Proposed flux estimator embedded in a current regulation 
expressed for a steady-state condition $(s=0)$ to highlight their main DC properties

$$
\begin{array}{r}
\left.\boldsymbol{G}_{d q, v}\right|_{s=0}=\left[\begin{array}{ll}
\frac{\omega_{\mathrm{me}}^{4}-\omega_{\mathrm{me}}^{2} \omega_{0}^{2}}{\left(\omega_{\mathrm{me}}^{2}-\omega_{0}^{2}\right)^{2}+4 \xi^{2} \omega_{\mathrm{me}}^{2} \omega_{0}^{2}} & -\frac{2 \xi \omega_{\mathrm{me}}^{3} \omega_{0}}{\left(\omega_{\mathrm{me}}^{2}-\omega_{0}^{2}\right)^{2}+4 \xi^{2} \omega_{\mathrm{me}}^{2} \omega_{0}^{2}} \\
\frac{2 \xi \omega_{\mathrm{me}}^{3} \omega_{0}}{\left(\omega_{\mathrm{me}}^{2}-\omega_{0}^{2}\right)^{2}+4 \xi^{2} \omega_{\mathrm{me}}^{2} \omega_{0}^{2}} & \frac{\omega_{\mathrm{me}}^{4}-\omega_{\mathrm{me}}^{2} \omega_{0}^{2}}{\left(\omega_{\mathrm{me}}^{2}-\omega_{0}^{2}\right)^{2}+4 \xi^{2} \omega_{\mathrm{me}}^{2} \omega_{0}^{2}}
\end{array}\right] \\
\left.\boldsymbol{G}_{d q, c}\right|_{s=0}=\left[\begin{array}{cc}
\frac{\omega_{0}^{4}-\omega_{\mathrm{me}}^{2} \omega_{0}^{2}+4 \xi^{2} \omega_{\mathrm{me}}^{2} \omega_{0}^{2}}{\left(\omega_{\mathrm{me}}^{2}-\omega_{0}^{2}\right)^{2}+4 \xi^{2} \omega_{\mathrm{me}}^{2} \omega_{0}^{2}} & \frac{2 \xi \omega_{\mathrm{me}}^{3} \omega_{0}}{\left(\omega_{\mathrm{me}}^{2}-\omega_{0}^{2}\right)^{2}+4 \xi^{2} \omega_{\mathrm{me}}^{2} \omega_{0}^{2}} \\
-\frac{2 \xi \omega_{\mathrm{me}}^{3} \omega_{0}}{\left(\omega_{\mathrm{me}}^{2}-\omega_{0}^{2}\right)^{2}+4 \xi^{2} \omega_{\mathrm{me}}^{2} \omega_{0}^{2}} & \frac{\omega_{0}^{4}-\omega_{\mathrm{me}}^{2} \omega_{0}^{2}+4 \xi^{2} \omega_{\mathrm{me}}^{2} \omega_{0}^{2}}{\left(\omega_{\mathrm{me}}^{2}-\omega_{0}^{2}\right)^{2}+4 \xi^{2} \omega_{\mathrm{me}}^{2} \omega_{0}^{2}}
\end{array}\right]
\end{array}
$$

Both matrices in (18) have non-infinite elements for $\xi \neq 0$ and $\omega_{0} \neq$ 0 , each representing a DC gain in the $d q$ reference frame. No matrix element diverges to infinite, as it would have happened in the case of an integral action for a DC component. This result is used in Section 3.2 to prove that (16) cannot converge to the actual flux without the use of an integral action, which is then added in the proposed estimator (17).

\subsection{Inductances and magnets flux sensitivity}

The sensitivity of the proposed estimator against variations of $L_{d}, L_{q}$ and $\lambda_{\mathrm{mg}}$ is a consequence of the results of Section 3.1. Considering the formula (16), the following equation holds

$$
\begin{aligned}
\hat{\boldsymbol{\lambda}}_{d q}= & \left(\boldsymbol{I}+\boldsymbol{G}_{d q, c}\right)^{-1} \boldsymbol{G}_{d q, v} \hat{\boldsymbol{\lambda}}_{d q, v} \\
& +\left(\boldsymbol{I}+\boldsymbol{G}_{d q, c}\right)^{-1} \boldsymbol{G}_{d q, c} \hat{\boldsymbol{\lambda}}_{d q, c}+\left(\boldsymbol{I}+\boldsymbol{G}_{d q, c}\right)^{-1} \boldsymbol{G}_{d q, c} \boldsymbol{\lambda}_{d q}
\end{aligned}
$$

where $\boldsymbol{I}$ is the identity matrix. Since $\boldsymbol{G}_{d q, c}$ does not contain any integral action, according to (18), the matrix $\left(\boldsymbol{I}+\boldsymbol{G}_{d q, c}\right)^{-1} \boldsymbol{G}_{d q, c}$ will not be an identity one for $s=0$ and the flux linkage estimation in (16) will not converge with zero steady-state error. In other words, the following cases are envisaged:

- if $\Delta \boldsymbol{\lambda}_{d q} \neq 0$, then $\boldsymbol{\lambda}_{d q} \neq \hat{\boldsymbol{\lambda}}_{d q}$ at steady state and the estimation has not yet converged to the actual flux linkage value;

- if $\Delta \boldsymbol{\lambda}_{d q}=0$, then expression (16) turns into the (11) which gives a wrong estimation, since the current model is not correct by initial hypothesis.

On the other hand, the proposed estimator in (17) leads to

$$
\begin{aligned}
\hat{\boldsymbol{\lambda}}_{d q} & =\frac{s K_{p}+K_{i}}{s+s K_{p}+K_{i}} \boldsymbol{\lambda}_{d q}+\frac{s}{s+s K_{p}+K_{i}} \boldsymbol{G}_{d q, c} \hat{\boldsymbol{\lambda}}_{d q, c} \\
& +\frac{s}{s+s K_{p}+K_{i}} \boldsymbol{G}_{d q, v} \hat{\boldsymbol{\lambda}}_{d q, v}
\end{aligned}
$$

In steady-state conditions $(s=0)$, the (20) returns $\hat{\boldsymbol{\lambda}}_{d q}=\boldsymbol{\lambda}_{d q}$. This implies that any change of $L_{d}, L_{q}$ or $\lambda_{\text {mg }}$ will be compensated by the estimator (20), zeroing the sensitivity of the proposed scheme to magnetic parameters changes, within the limits of the accuracy of $\Delta \boldsymbol{\lambda}_{d q}$, of course.

\subsection{Stator resistance sensitivity}

The proposed estimator is still affected by variations of the stator resistance, which is present in the voltage model (5) and in the compensation term (15). The sensitivity analysis should consider the closed-loop nature of the proposed estimator in combination with the FOC, as depicted in Fig. 1.

The mathematical expression of the stator resistance sensitivity for the scheme of Fig. 1 was obtained as follows: first, the voltages as

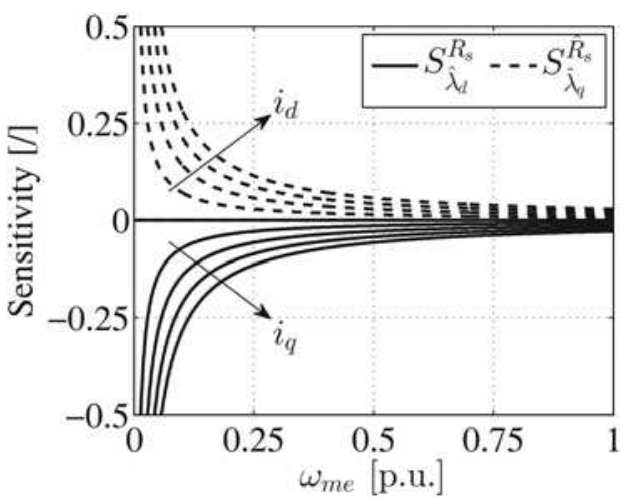

Fig. 2 Sensitivity functions of the estimated flux on $d$ and $q$ axes

output of the current regulation loop have been assessed, and an expression for the phase motor currents was found, based on the calculated voltages. After that, an evaluation of $\Delta \boldsymbol{\lambda}_{d q}$ was performed. Finally, an expression for $\hat{\boldsymbol{\lambda}}_{d q}$ was found, whose derivative with respect to $\hat{R}_{s}$ gives indications on the estimator sensitivity. The following expressions were found

$$
\begin{aligned}
& \left.\frac{\mathrm{d} \hat{\lambda}_{d}}{\mathrm{~d} \hat{R}_{s}}\right|_{s=0}=-\frac{i_{q}}{\omega_{\mathrm{me}}} \\
& \left.\frac{\mathrm{d} \hat{\lambda}_{q}}{\mathrm{~d} \hat{R}_{s}}\right|_{s=0}=\frac{i_{d}}{\omega_{\mathrm{me}}}
\end{aligned}
$$

According to the (21), the sensitivity functions $S_{\hat{R}_{s}}^{\hat{\lambda}_{d}}=\left(\mathrm{d} \hat{\lambda}_{d} / \mathrm{d} \hat{R}_{s}\right) R_{s} / \lambda_{n}$ and $S_{\hat{R}_{s}}^{\hat{\lambda}_{q}}=\left(\mathrm{d} \hat{\lambda}_{q} / \mathrm{d} \hat{R}_{s}\right) R_{s} / \lambda_{n}$ were calculated, where $R_{s}$ and $\lambda_{n}$ are the nominal stator resistance and the nominal flux linkage value obtained from Table 2. A graphical representation of the sensitivity (21) is shown in Fig. 2.

The sensitivity of the flux linkage estimates to stator resistance (21) is related to the compensating term (15). Actually, the voltage model in (5) contains a stator resistance dependence, which in theory could have been compensated with the proposed $\Delta \boldsymbol{\lambda}_{d q}$, if the latter had not a stator resistance dependence itself, as shown in (15). In other words, the estimator sensitivity in (21) is a result of the sensitivity of (15), not of the sensitivity of (5). It is worth to underline that stator resistance mismatches cause larger estimation errors as the speed goes down below the crossover frequency $\omega_{0}$, where anyway the current model starts to weight more than the voltage model in the flux linkage estimation.

\section{Simulation results}

The simulation results of the flux estimator proposed in Section 2.3 are compared with the original flux estimator of [21] and illustrated in Section 2.2. The parameters of the isotropic synchronous motor and the flux estimator used in the simulations are shown in the Appendix (Tables 2 and 3, respectively). To test the behaviour of the flux estimators with an incorrect current model, the estimated parameters are set with the following values

$$
\begin{aligned}
\hat{L}_{d} & =1.3 \cdot L_{d} \\
\hat{L}_{q} & =1.3 \cdot L_{q} \\
\hat{\lambda}_{\mathrm{mg}} & =1.3 \cdot \lambda_{\mathrm{mg}} \\
\hat{R}_{s} & =R_{s}
\end{aligned}
$$

The simulation test is performed by applying a load torque step equal to the nominal torque $\tau_{N}$ at a mechanical speed of $239 \mathrm{rpm}$. This speed, with the cut-off frequency $\omega_{0}$ shown in Table 3 , guarantees 

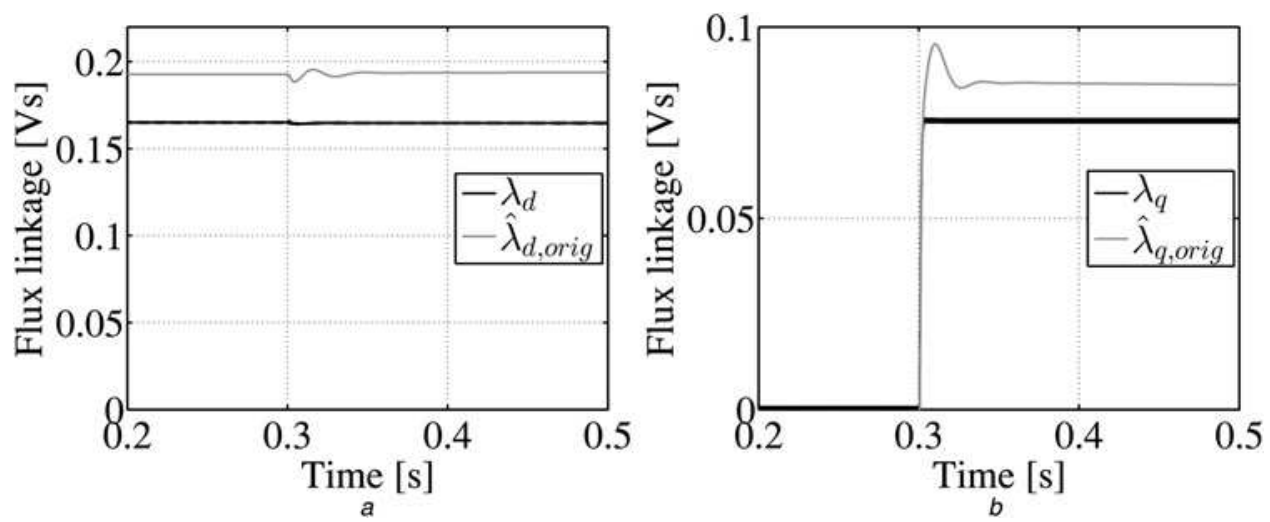

Fig. 3 Original flux estimation

$a$ In $d$ axis

$b$ In $q$ axis
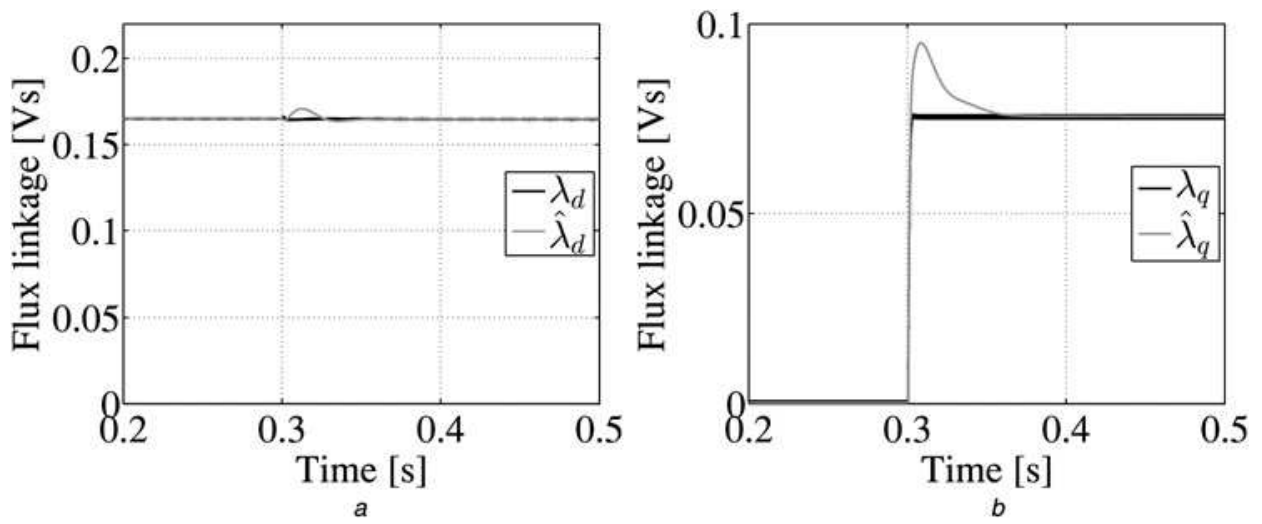

Fig. 4 Proposed flux estimation

$a$ In $d$ axis

$b \operatorname{In} q$ axis

that the flux estimation depends only on the current model output. Fig. 3 shows the flux estimation obtained from the original estimator $\left(\hat{\lambda}_{d \text {,orig }}\right.$ and $\left.\hat{\lambda}_{q \text {,orig }}\right)$ compared with the actual flux linkage $\left(\lambda_{d}\right.$ and $\left.\lambda_{q}\right)$. In Fig. 4 the actual flux linkage is compared with the proposed flux estimation $\left(\hat{\lambda}_{d}\right.$ and $\hat{\lambda}_{q}$ ). The simulation confirms that the proposed flux estimator returns an error-free flux linkage estimation.

An important aspect investigated in simulations relates to the tuning of the PI regulator in the flux linkage estimation. Together with the current PI regulator, the proposed flux estimator contributes to generate the voltage reference for the inverter, while exploiting at the same time the integral part of the current PI regulators themselves (see Fig. 1). Thus, if the bandwidth of the flux estimator loop is lower with respect to the current loop one, the two loops will not interfere. Furthermore, an accurate compensation of the coupling terms in (2) requires the flux estimator loop to be faster than the speed control loop. The tuning of the PI coefficients in the (17) for simulations and experimental tests confirms the aforementioned constraints on the flux observer loop bandwidth.

\section{Experimental results}

\subsection{Test bench description}

The proposed flux estimator has been tested on the same PM machine simulated in Section 4. The machine is a part of the set-up shown in Fig. 5, where the FOC algorithm, the flux estimator and the current/position/DC-bus voltage acquisition

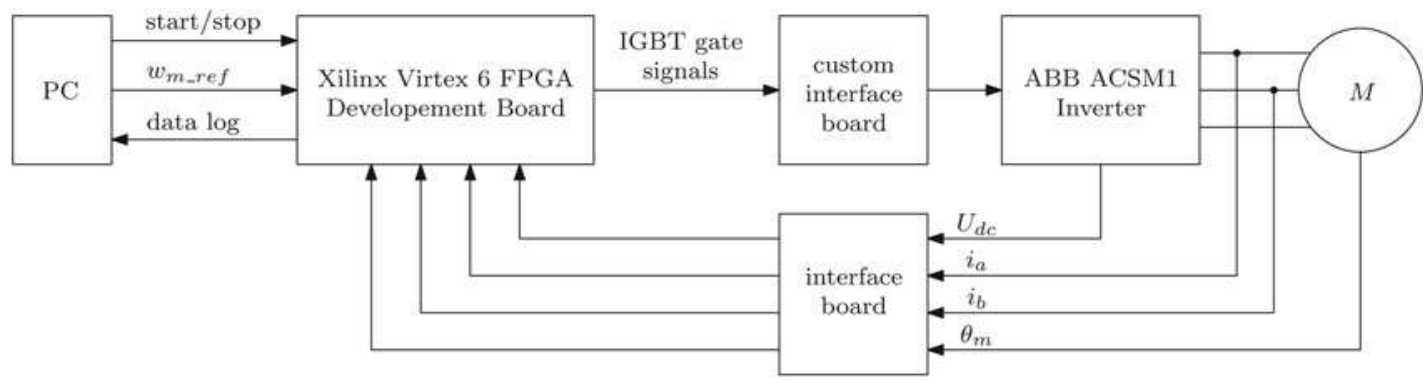

Fig. 5 Block diagram of the all-on-chip FPGA solution 


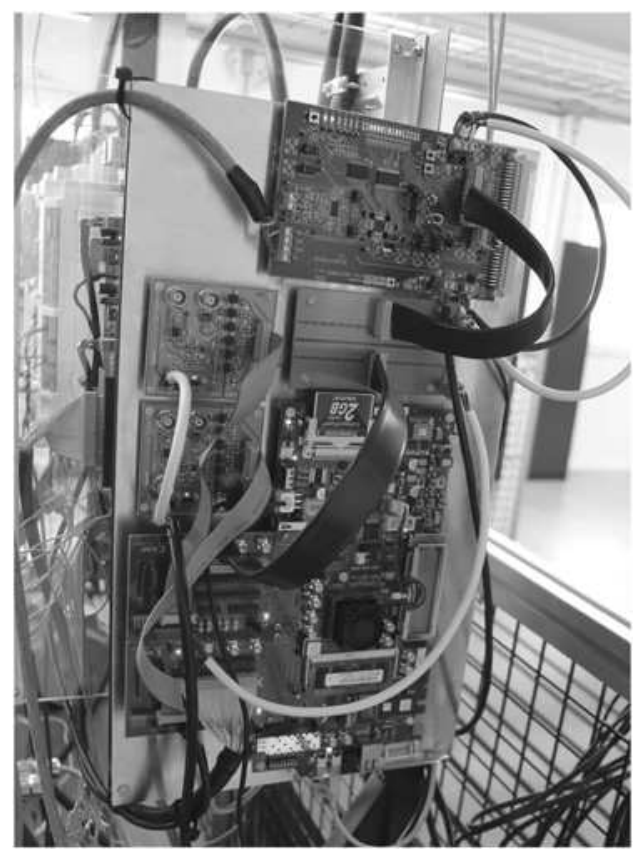

Fig. 6 FPGA board with its signal interface boards

process have been implemented on a Xilinx Virtex 6 FPGA development board, with a clock frequency of $200 \mathrm{MHz}$ (see Fig. 6). The board was used for prototyping purposes only. The sampling process is synchronous with the implemented PWM at $10 \mathrm{kHz}$. From the analog signals of a conventional resolver and a resolver-to-digital conversion, the speed is also calculated in the development board by differentiating two consecutive values of the sampled position. The board is connected to an ABB ACSM1 inverter by means of a custom interface board. The PC block present in the block schematic is for interface and data logging only. The whole control system is synthesised on the FPGA chip, without any other microprocessor. Fig. 7 shows the back-to-back configuration of two synchronous motors used in the experiments, of which one is the motor under test and the other is the load machine. A torque meter is connected on the shaft.

\subsection{FPGA implementation}

Fig. 8 shows a time diagram of the control routine implementation, highlighting the effort of task parallelisation which can be truly achieved only on FPGAs (design methodologies can be found for

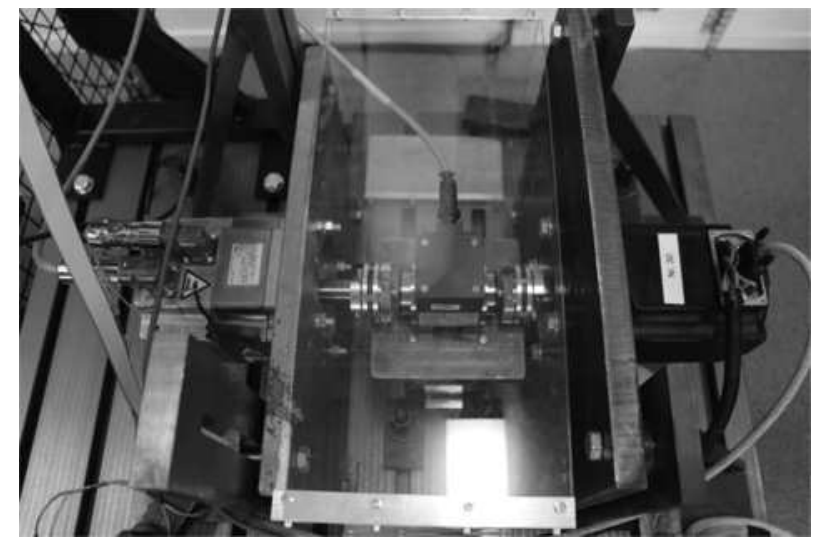

Fig. 7 Test motor (on the left-hand side) and load motor (on the right-hand side) example in [24]). The conventional FOC computation (white background blocks) is performed in parallel to the flux estimation computation (grey background blocks). Each block performs its calculations as fast as its required inputs are available from the previous calculations. The execution speed of the entire routine is in the order of $2 \mu \mathrm{s}$ excluding the time required for the current, DC-bus voltage and speed sensor measurements reading.

FPGA usage is a consolidated trend for next-generation drives, nevertheless their use requires skills that somewhat recall the very first assembly programs. Since the FPGA hardware resources have a close relation with cost, some savings are obtained by a careful implementation. For example, in Fig. 8, the 'SVM' block performs a conventional space vector modulation, which requires a division by the DC-bus voltage $U_{\mathrm{dc}}$. The division is not executed in the FPGA, substituted by the block ' $U_{\mathrm{dc}}$ processing' where $U_{\mathrm{dc}}$ is approximated with integers in the form of $U_{\mathrm{dc}}=2^{n} / m$, with $n \in \mathbb{N}$. In this way, the division is performed with a multiplication by $m$ and a right-shift of $n$ bits. It is also worth to note that all constants and internal control variables have been scaled to integer representation with no decimal point, according to the desired resolution. Depending on the expected range of the variables, the number of bits for each variable representation was set. Ranges from 6 to 32 bits have been used.

Most of the blocks in Fig. 8 are self-explaining. Hereafter will be given a short description of the less clear ones. The 'PI $i_{d q}$ and decoupling' block receives the current references from the ${ }^{i d q}$ speed regulation, the measured currents and the $d q$-axis decoupling terms for performing a conventional current regulation with decoupling of the axes applied at the regulators output. The 'SVM delay compensation' block shifts forward the phase of the voltage reference vector of the same amount of phase delay introduced by the inverter. In this work, the space-vector modulation is symmetric and updates the voltage reference vector each sample time, meaning that the delay between sampled currents and actual voltage generation is approximated as 1.5 times the PWM sample time. The technique used for the phase advance is described in [25]. Among the flux estimation blocks, the (17) (excluding the $\Delta \boldsymbol{\lambda}_{d q}$ term) is implemented in the 'Merge of voltage and current models' block, whereas the ' $\Delta \boldsymbol{\lambda}_{d q}$ ' block calculates the (15) by also setting a lower limit for $\left|\omega_{m}\right|$, to avoid divisions by zero. If the mechanical speed is in the interval $[-2 \mathrm{rad} / \mathrm{s}, 2 \mathrm{rad} / \mathrm{s}]$, no compensation is calculated and used in the flux estimation. The ' $d q$ decoupling term' block calculates the estimation of last term in the (2), by using the estimated fluxes. The 'IGBT pulse generation' creates the gate commands from the duty cycles computed in the SVM block. It is worth to note that all blocks were implemented with VHDL code, and interconnected with the help of Xilinx System Generator. This choice was adopted to ease the debugging of the system and the monitoring of the control variables.

As it is well known, logic resources on the FPGA are grouped in slices to create configurable logic blocks. Each slice of the Virtex 6 family contains 4 LUTs and 8 flip-flops and represents the smaller reconfigurable block. The compiler assigns the functionalities of the blocks, including the LUTs, according to the user code. As an example, the slices can use their LUTs as distributed 64-bit RAMs or as 32-bit shift registers. As a term of comparison and, possibly, as an aid for the design, Table 1 reports the FPGA hardware resources required by each part of the control algorithm.

Although the complete implementation of the drive on the FPGA chip was successful, such approach proved to be quite difficult to debug. A master-slave solution, where a microprocessor could be used for some operations, may seem a more reasonable solution today to cut the time-to-market of the product. Nevertheless, it is expected that the task of FPGA programming and debugging will become easier in the future, allowing a faster implementation of the proposed approach. Moreover, the efforts profused to squeeze the algorithm in the FPGA paid back in an extremely fast execution of the algorithm, potentially leaving a large amount of time to DSPs to perform other relevant tasks (trajectory calculation, parallel synchronisation with other drives, communication with other drives or external devices etc.). 


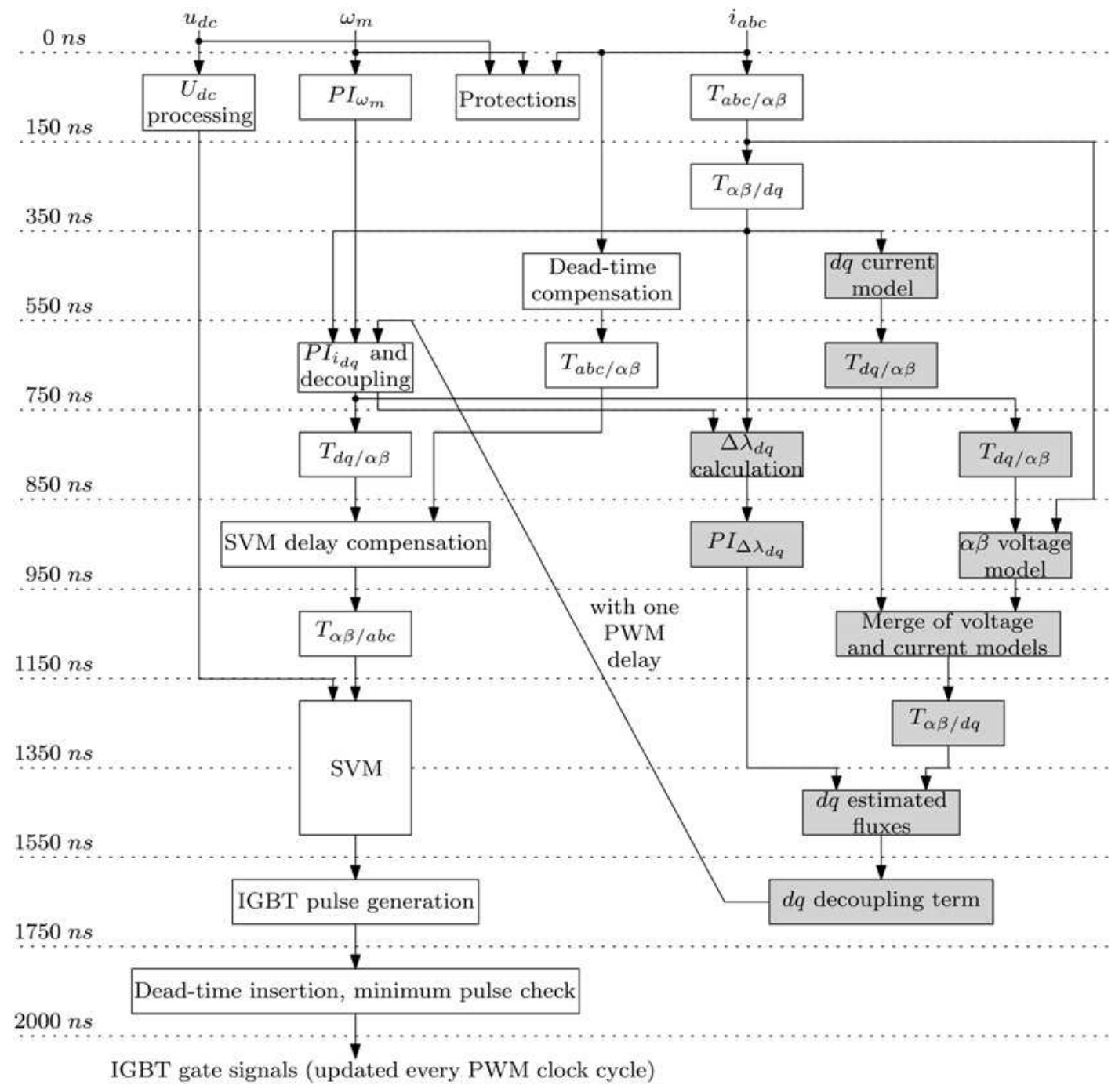

Fig. 8 Time execution of the algorithm on the FPGA device

\subsection{Transient behaviour}

Fig. 9 shows the motor behaviour when a speed reference step and a load torque step are applied. The initial speed reference is $183 \mathrm{rpm}$ while the final one is $732 \mathrm{rpm}$. The FOC forces to zero the $d$-axis current reference because with an isotropic motor no reluctance torque is required, and field weakening is not applied. The motor is initially not loaded and the load torque step value is equal to the nominal one (see Table 2 for the machine data). After both variations the control loop continues to operate properly, the motor speed following its reference value (Fig. 9a). The load torque step has requested the nominal current from the machine, saturating the output of the speed regulator (current reference) which was initially fixed to the nominal current of the motor (see Table 2), and slowing the speed recovery. The $q$-axis current changes according to Figs. $9 b-d$ zoom into the $q$-axis current transients.

Fig. 10 shows the estimated flux linkage $\hat{\boldsymbol{\lambda}}_{\boldsymbol{d} q}$ with respect to the actual flux linkage $\boldsymbol{\lambda}_{d q}$, the latter obtained by previous off-line

Table 1 FPGA hardware usage

\begin{tabular}{lcc}
\hline Function & $\begin{array}{c}\text { Registers }(\% \text { of } \\
\text { available) }\end{array}$ & $\begin{array}{c}\text { LUTs (\% of } \\
\text { available) }\end{array}$ \\
\hline flux estimator & $1035(0.3 \%)$ & $6322(4.2 \%)$ \\
field oriented control & $1602(0.5 \%)$ & $7022(4.7 \%)$ \\
I/O conditioning and & $1206(0.4 \%)$ & $953(0.6 \%)$ \\
SVM & & \\
\hline
\end{tabular}

measurements of the magnetic characteristics of the machine (see again Table 2). The profile of the estimated flux on the q-axis is zoomed in Figs. $10 c$ and $d$, showing a good tracking of the actual flux during no load and full load conditions, even in presence of saturation of the speed regulator.

The behaviour of the system when the current model is predominant are shown in Figs. 11 and 12. In these tests, the maximum current output from the PI speed regulator was fixed to the zero-speed maximum current of the motor (equal to $4.7 \mathrm{~A}$, see Table 2). Fig. 11 shows an overloading condition case of $119 \%$ of the nominal torque at low speed, with the flux estimation curves following the actual fluxes coherently. Fig. 12 shows the case where the rotor is blocked by an excessive load $(127 \%$ of the nominal torque), and subsequently released. During the blocked-rotor interval, the $i_{q}$ current reaches the maximum level of 4.7 A (equal to the zero-speed maximum current from the motor datasheet). After the release, the speed regulation returns back to its normal operation. The estimated fluxes follow very well the actual fluxes during both the blocked-rotor condition and after the load release.

\subsection{Experimental parametric sensitivity}

In this section, the proposed flux estimation has been tested against variations of the machine parameters, by changing the values of $\hat{L}_{d}$, $\hat{L}_{q}, \hat{\lambda}_{\text {mg }}$ and $\hat{R}_{s}$ during the motor operation and recording the behaviour of the flux estimation itself. These tests aim to resemble the case where parameters are not perfectly known because of an 


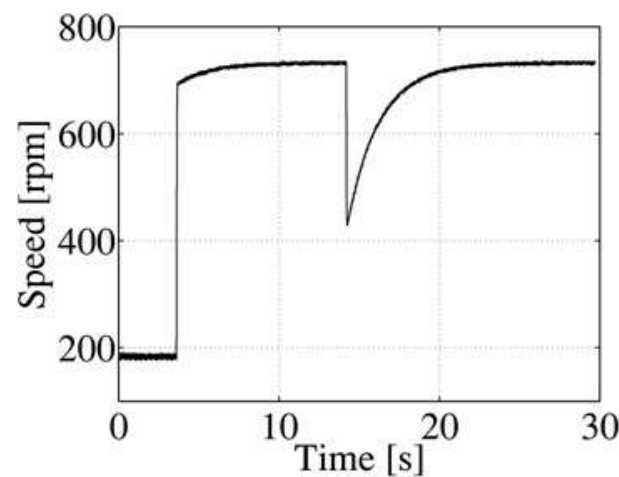

a

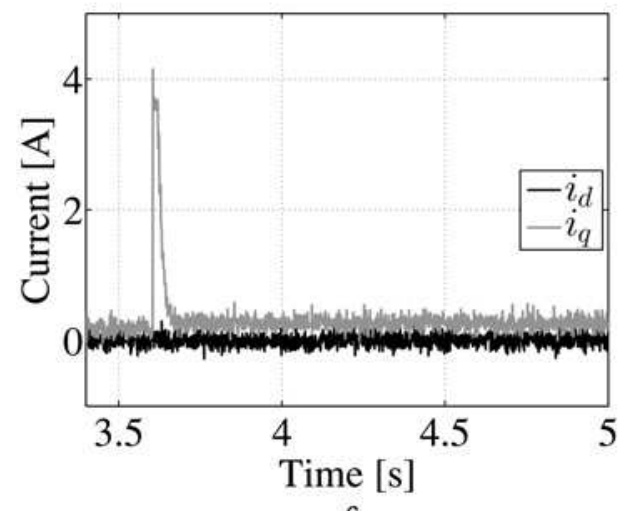

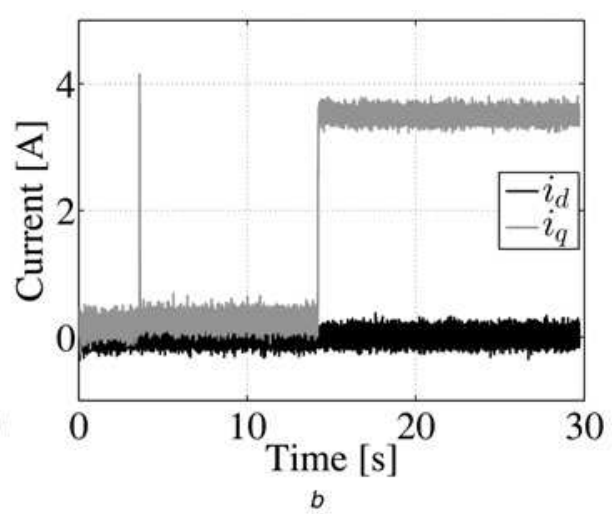

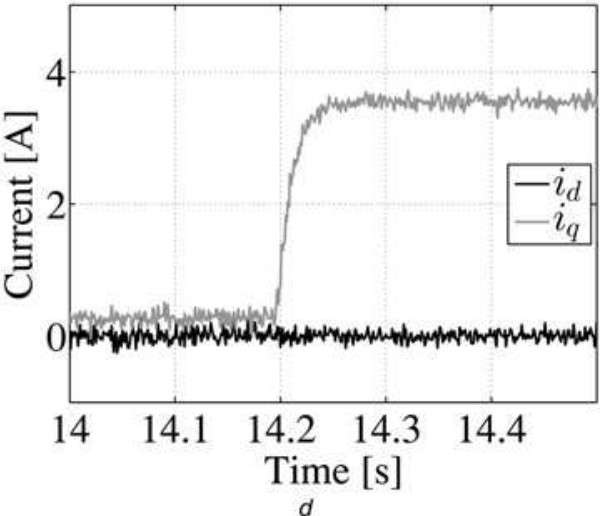

Fig. 9 Speed and load torque transients

$a$ Mechanical speed

$b d q$ currents

$c$ and $d$ are a zoom of $b$
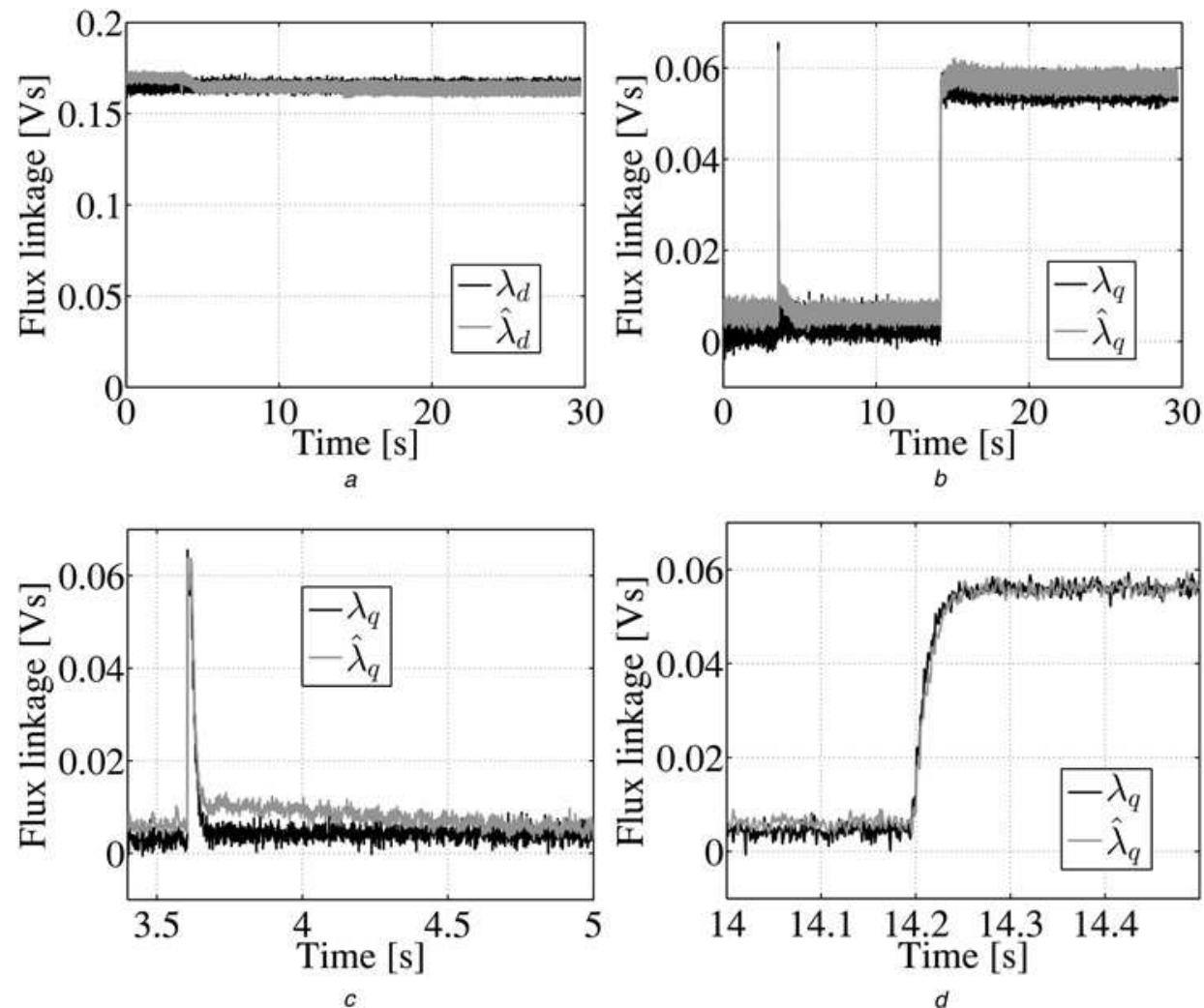

Fig. 10 Flux linkage estimation in

a $d$ axis

$b q$ axis during speed and load torque transients

$c$ and $d$ are a zoom of $b$ 

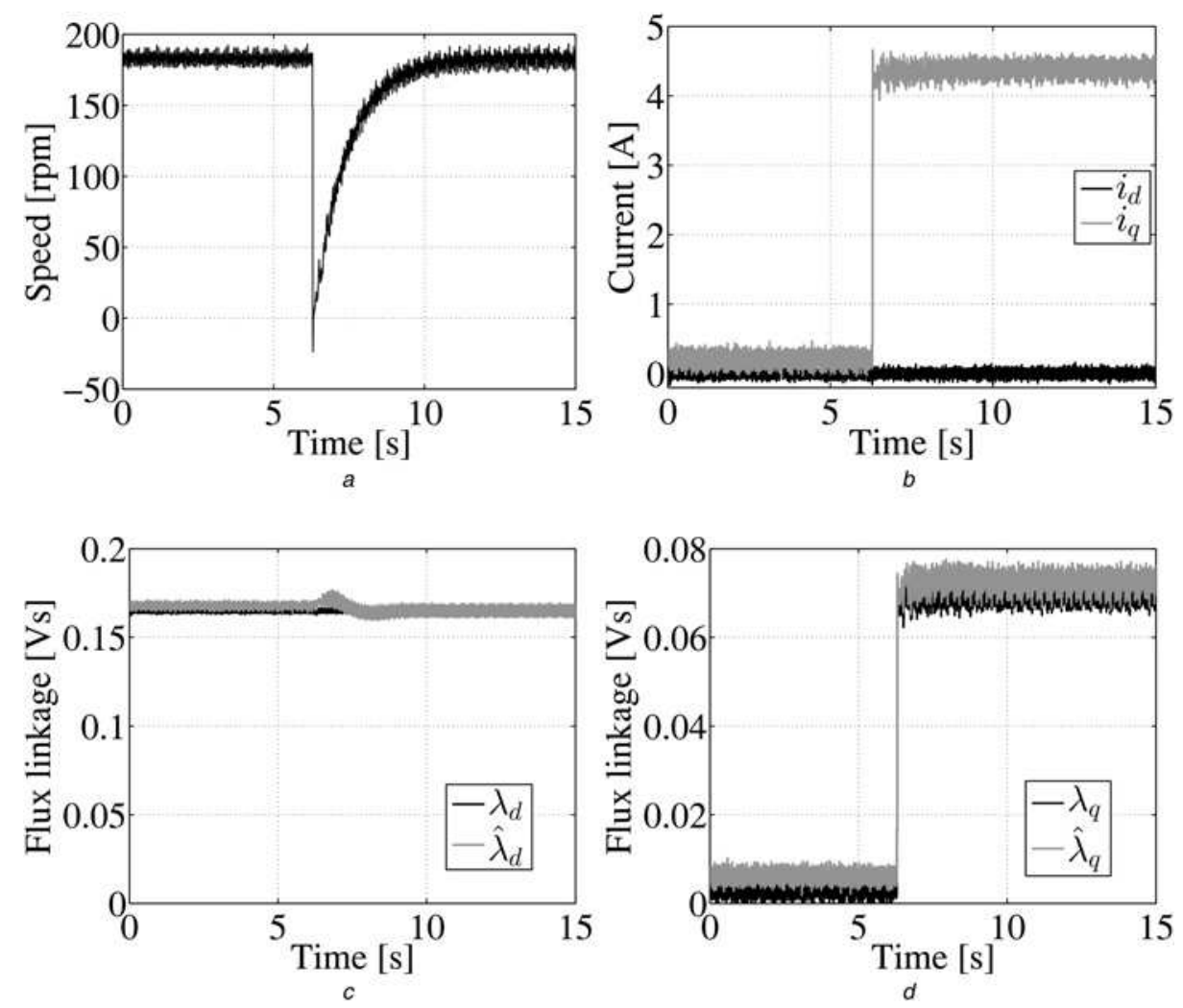

Fig. 11 Torque overload of $119 \%$

$a$ Mechanical speed

$b d q$ currents

$c d$-axis actual and estimated flux

$d q$-axis actual and estimated flux
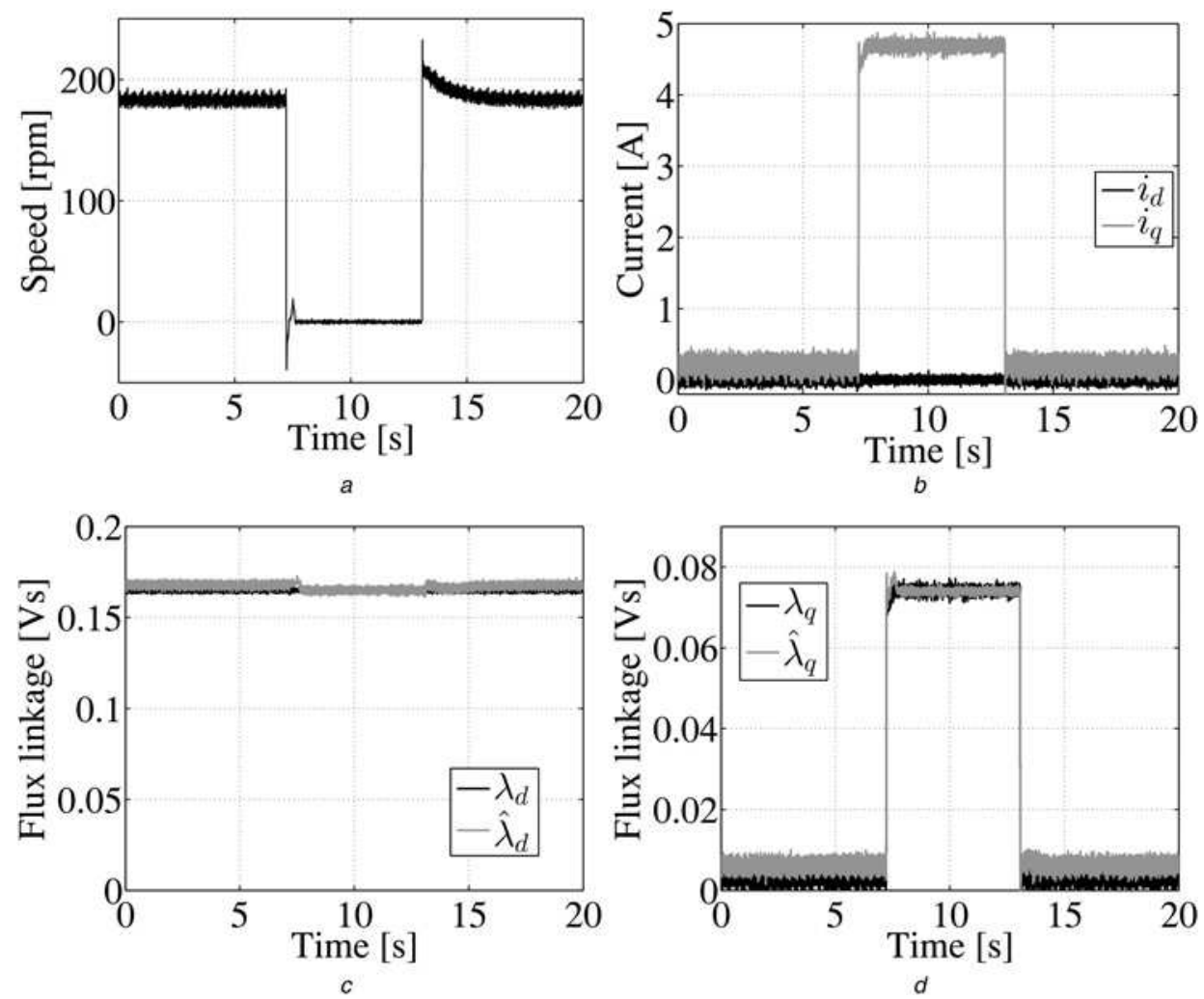

Fig. 12 Temporary blocked rotor condition because of excessive load

$a$ Mechanical speed

$b d q$ currents

$c d$-axis actual and estimated flux

$d q$-axis actual and estimated flux 

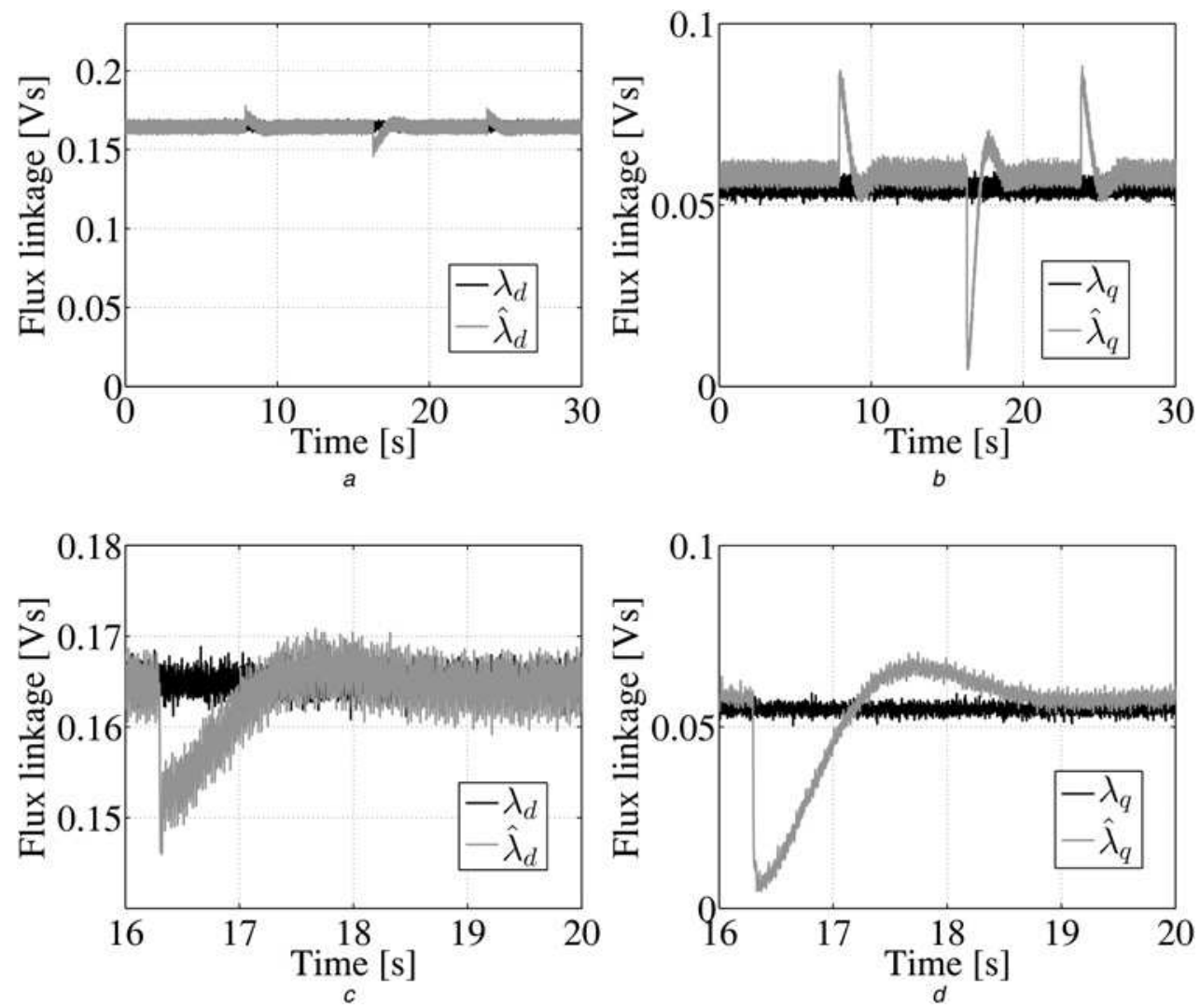

Fig. 13 Actual and estimated flux with $\hat{L}_{q}$ variations

a $d$-axis flux

$b q$-axis flux

$c$ and $d$ are a zoom of $a$ and $b$

incorrect commissioning or a variation because of temperature or different operating points. The reference value of the machine parameters have been obtained by preliminary tests following the procedure described in $[5,7]$. The procedure exploits a load machine connected back-to-back with the machine under test. The load machine keeps a constant speed, while the machine under test is only current-regulated. The desired levels of $i_{d}$ and $i_{q}$ are applied, recording the corresponding voltage. From the knowledge of the stator resistance, the voltages and the currents, $\lambda_{d}$ and $\lambda_{q}$ (and thus $L_{d}$ and $L_{q}$ ) as function of $i_{d}$ and $i_{q}$ are indirectly calculated.

Fig. 13 shows the estimator behaviour when $\hat{L}_{q}$ varies of $30 \%$ with respect to the actual $L_{q}$ value. The load torque applied is $3.05 \mathrm{Nm}$ $\left(82 \%\right.$ of $\left.\tau_{N}\right)$ and the reference speed is maintained constant at 183 $\mathrm{rpm}$. This speed value guarantees that the estimator operates only with the current model and therefore it is influenced by $\hat{L}_{q}$ variations. In details, the test of Fig. 13 consists of the following steps:

- initial condition: $\hat{L}_{q}=L_{q}$

- first step: $\hat{L}_{q}=1.3 \cdot L_{q}$

- second step: $\hat{L}_{q}=0.7 \cdot L_{q}$

- third step: $\hat{L}_{q}=L_{q}$

When $\hat{L}_{q} \neq L_{q}$, the flux current model gives a wrong flux estimation, but the additional term $\Delta \boldsymbol{\lambda}_{d q}$ processed by the PI
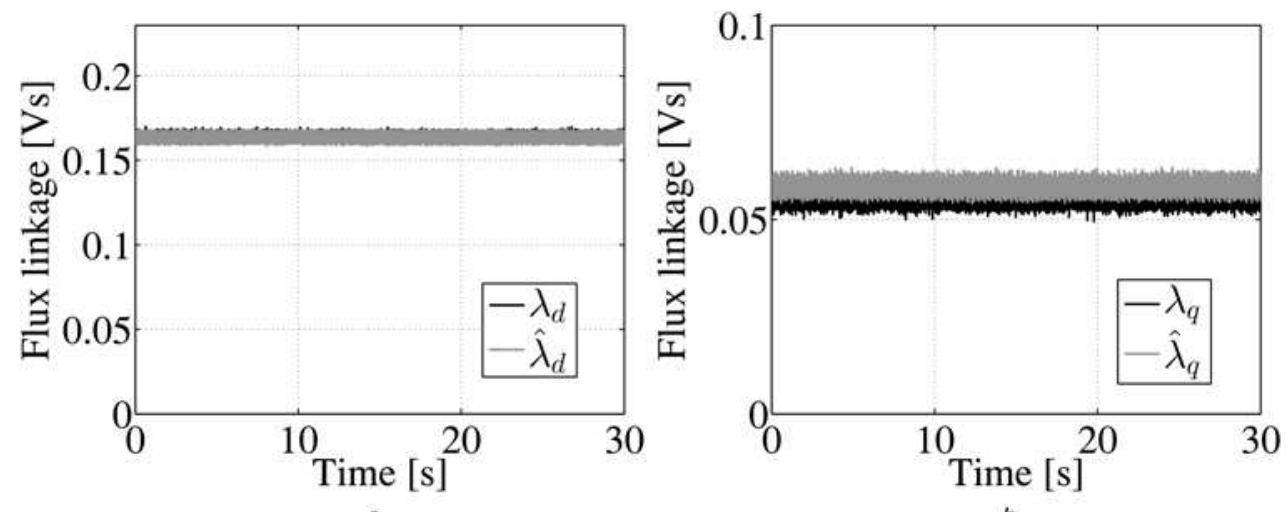

Fig. 14 Actual and estimated flux with $\hat{L}_{d}$ variations

$a d$-axis flux

$b q$-axis flux 

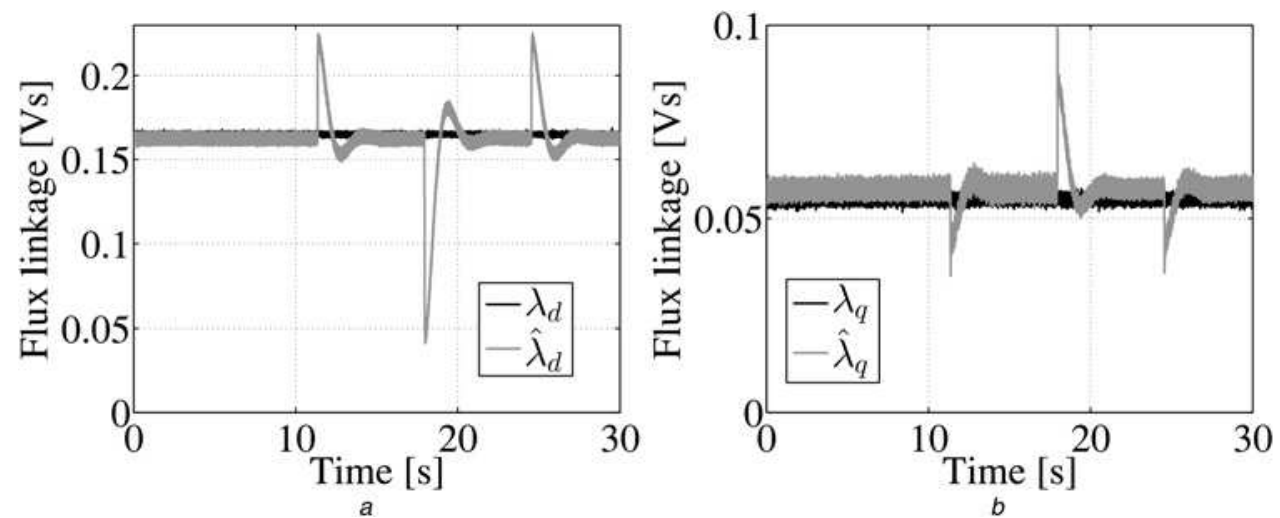

Fig. 15 Actual and estimated flux with $\hat{\lambda}_{\text {mg }}$ variations

$a d$-axis flux

$b q$-axis flux
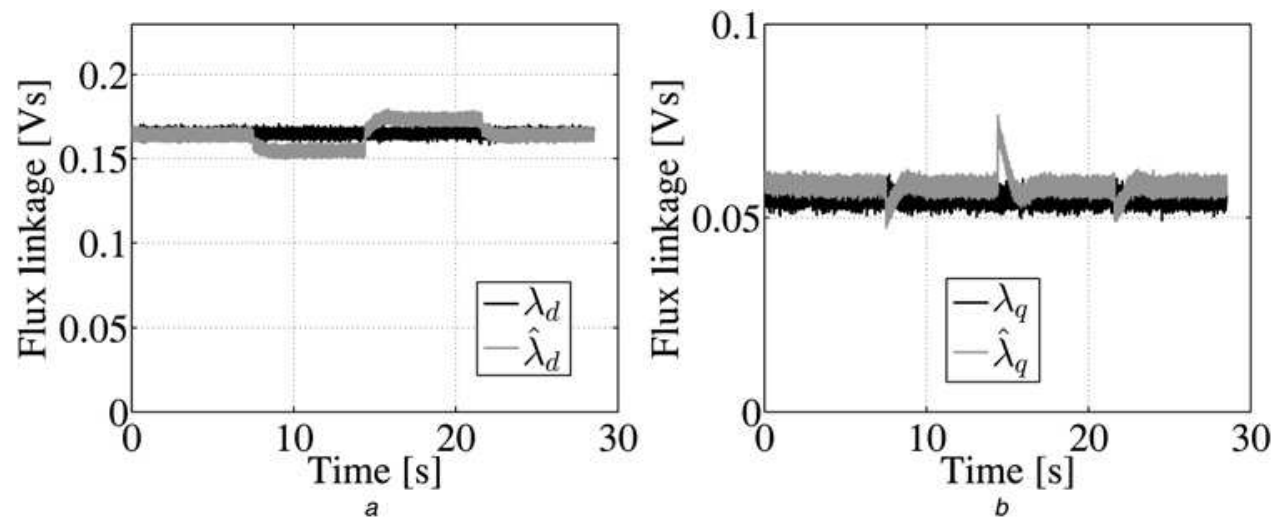

Fig. 16 Actual and estimated flux with $\hat{R}_{s}$ variations

$a d$-axis flux

$b q$-axis flux

regulator brings back the estimation to the correct output, after a short transient.

The same test has been performed by imposing $\hat{L}_{d}$ variations. With an $i_{d}$ forced to be zero by the current controller, the variations of $\hat{L}_{d}$ do not affect the flux estimation (see Fig. 14).

Fig. 15 shows the flux linkage estimation when $\hat{\lambda}_{\mathrm{mg}}$ varies of $\pm 30 \%$ with respect to $\lambda_{\mathrm{mg}}$ value. The speed reference and the load torque are the same of the previous two experiments.

The last test regards a $\hat{R}_{s}$ variation of $\pm 40 \%$. This parameter is present in the voltage model and not in the current model. Thus, the test has been done with a different speed reference $(549 \mathrm{rpm})$ which makes the flux estimation dependent on the voltage model output. The results are shown in Fig. 16 where the load torque applied has been maintained the same as the previous tests.

As previously analysed in Section 3, the term $\Delta \boldsymbol{\lambda}_{d q}$ is not able to fully compensate the error as in the previous tests. In particular, since $i_{d}$ is forced to zero by the control, variations of $\hat{R}_{s}$ do not have any influence on the steady-state values of $\lambda_{q}$, according to the second of the (21). On the other hand, with non-zero $i_{q}$, the variations of $\hat{R}_{s}$ influence negatively the $d$-axis component of $\Delta \boldsymbol{\lambda}_{d q}$, as described in the first of the (21). Both results are numerically valid if compared with the theoretical expressions in (21).

If the field weakening technique is implemented, it is expected that errors in the flux estimation are caused by stator resistance variations only, since the current model is not used at high speeds. Nevertheless, at high speed bemfs are normally much higher than the resistive drops, which are then expected to cause negligible effects.

\section{Conclusions}

A flux linkage estimator based on a combined voltage and current models was analysed, considering the modifications required to obtain zero-error convergence of the estimation at steady-state. The theoretical development was accompanied with an extensive parameter sensitivity analysis. Simulation and experimental tests were performed on an isotropic synchronous machine, to validate the proposed algorithm.

The conventional FOC and the proposed flux estimation schemes were implemented on a FPGA-based control board. The implementation did not use any microprocessor and exploited parallelisation of the calculations, reaching a very fast execution time of the algorithm.

The performances of the flux estimator were shown during speed and load torque transients, confirming the robustness of the approach. Further refinements of the work will encompass the development of an on-line stator resistance estimation, which is still affecting the overall output of the flux estimation if large mismatches occur.

\section{Acknowledgments}

The Authors would like to thank Dr. Riccardo Antonello for his contribution in the development of the theoretical analysis of the estimator. 


\section{References}

1 Park, Y., Sul, S.-K.: 'Sensorless control method for PMSM based on frequency-adaptive disturbance observer', IEEE J. Emerging Sel. Top. Power Electron., 2014, 2, (2), pp. 143-151

2 Specht, A., Wallscheid, O., Bocker, J.: 'Determination of rotor temperature for an interior permanent magnet synchronous machine using a precise flux observer'. Proc. of Power Electronics Conf. (ECCE-ASIA 2014), Bristol, UK, June 2014, pp. $1501-1507$

3 Vagati, A., Pastorelli, M., Franceschini, G., Drogoreanu, V. 'Flux-observer-based high-performance control of synchronous reluctance motors by including cross saturation', IEEE Trans. Ind. Appl., 1999, 35, (3), pp. 597-605

4 Rahman, K.M., Hiti, S.: 'Identification of machine parameters of a synchronous motor', IEEE Trans. Ind. Appl., 2005, 41, (2), pp. 557-565

5 Cintron-Rivera, J.G., Babel, A.S., Montalvo-Ortiz, E. E., Foster, S.N., Strangas, E.G. 'A simplified characterization method including saturation effects for permanent magnet machines'. Proc. of the 20th IEEE Int. Conf. on Electrical Machines (ICEM 2012), Marseille, France, 2-5 September 2012, pp. 837-843

$6 \mathrm{Li}, \mathrm{Z}, \mathrm{Li}, \mathrm{H}$. 'MTPA control of PMSM system considering saturation and cross-coupling'. Proc. of the 15th IEEE Int. Conf. on Electrical Machines and Systems (ICEMS 2012), Sapporo, Japan, 21-24 October 2012, pp. 1-5

7 Armando, E., Bojoi, R., Guglielmi, P., Pellegrino, G., Pastorelli, M.: 'Experimental identification of the magnetic model of synchronous machines', IEEE Trans. Ind. Appl., 2013, 49, (5), pp. 2116-2125

8 Kim, Y.-S., Sul, S.-K.: 'Torque control strategy of an IPMSM considering the flux variation of the permanent magnet'. Proc. of the 42nd IEEE Industry Application Conf. Annual Meeting (IAS 2007), New Orleans, Louisiana, USA, 23-27 September 2007, pp. 1301-1307

9 Kang, G., Lim, J., Nam, K., Ihm, H.-B., Kim, H.-G.: 'A MTPA control scheme for an IPM synchronous motor considering magnet flux variation caused by temperature'. Proc. of the 19th Annual IEEE Applied Power Electronics Conf. and Exposition (APEC 2004), Anaheim, California, USA, 22-28 February 2004, vol. 3, pp. 1617-1621

10 Babel, A.S., Cintron-Rivera, J.C., Strangas, E.G.: 'A multiple look-up table torque controller for improved performance of IPM machines'. Proc. of the IEEE Int. Electrical Mlachines and Drives Conf. (IEMDC 2013), Chicago, Illinois, USA, 12-15 May 2013, pp. 521-525

11 Hoang, K.D., Wang, J., Cyriacks, M., Melkonyan, A., Kriegel, K. 'Feed-forward torque control of interior permanent magnet brushless AC drive for traction applications'. Proc. of the IEEE Int. Electrical Mlachines and Drives Conf. (IEMDC 2013), Chicago, Illinois, USA, 12-15 May 2013, pp. 152-159

12 Holtz, J., Quan, J: 'Sensorless vector control of induction motors at very low speed using a nonlinear inverter model and parameter identification', IEEE Trans. Ind. Appl., 2002, 38, (4), pp. 1087-1095

13 Kellner, S.L., Piepenbreier, B.: 'Identification of ohmic stator resistance based on low frequency current signal injection in permanent magnet synchronous machines'. Proc. of the 14th International Power Electronics and Motion Control Conf. (EPE/PEMC 2010), Ohrid, R. Macedonia, 6-8 September 2010, pp. T5-221-T5-229

14 Boileau, T., Nahid-Mobarakeh, B., Meibody-Tabar, F.: 'On-line identification of PMSM parameters: model-reference vs EKF', Proc. of the 2008 IEEE Industry Applications Society Annual Meeting (IAS 2008), Edmonton, Alberta, Canada, 5-9 October 2008, pp. 1-8

15 Song, W., Shuang-shuang, S., Chao, C., Gang, Y., Zhi-jian, Q.: 'Identification of PMSM based on EKF and Elman neural network'. Proc. of the IEEE Int. Conf. on Automation and Logistics (ICAL 2009), Shenyang, China, 5-7 August 2009, pp. $1459-1463$

16 Ichikawa, S., Tomita, M., Doki, S., Okuma, S.: 'Sensorless control of permanent magnet synchronous motors using online parameter identification based on system identification theory', IEEE Trans. Ind. Electron., 2006, 53, (2), pp. 363-372

17 Piippo, A., Hinkkanen, M., Luomi, J.: 'Adaptation of motor parameters in sensorless PMSM drives', IEEE Trans. Ind. Appl., 2009, 45, (1), pp. 203-212

18 Mohamed, Y.-R., Lee, T.: 'Adaptive self-tuning MTPA vector controller for IPMSM drive system', IEEE Trans. Energy Convers., 2006, 21, (3), pp. 636-644
19 Niazi, P., Toliyat, H.A.: 'Online parameter estimation of permanent-magnet assisted synchronous reluctance motor', IEEE Trans. Ind. Appl., 2007, 43, (2), pp. 609-615

20 Jeong, Y., Sul, S.-K.: 'Adaptive flux observer with on-line inductance estimation of an IPMSM considering magnetic saturation'. Proc. of the IEEE 36th Power Electronics Specialists Conf. (PESC), Recife, Brazil, 12-18 June 2005, pp. 2467-2473

21 Yoo, A., Sul, S.-K. 'Design of flux observer robust to interior permanent-magnet synchronous motor flux variation', IEEE Trans. Ind. Appl., 2009, 45, (5), pp. $1670-1677$

22 Vaez-Zadeh, S., Zamanifar, M., Soltani, J.: 'Nonlinear efficiency optimization control of IPM synchronous motor drives with online parameter estimation'. Proc. of the 6th IEEE Power Electronics Specialists Conf. (PEMD 2006), Jeju, South Korea, 18-22 June 2006, pp. 1-6

23 Peretti, L., Zigliotto, M.: 'Combined current/voltage model with inductance mismatch compensation for on-line estimation of flux linkages in permanent magnet and synchronous reluctance motor drives'. Proc. of the Sixth Power Electronics, Machines and Drives Conf. (PEMD 2012), Bristol, UK, 27-29 March 2012, pp. 1-6

24 Naouar, M.-W., Monmasson, E., Naassani, A.A., Slama-Belkhodja, I., Patin, N.: 'FPGA-based current controllers for AC machine drives - a review', IEEE Trans. Ind. Electron., 2007, 54, (5), pp. 1907-1925

25 Bolognani, S., Peretti, L., Zigliotto, M.: 'Repetitive-control-based self-commissioning procedure for inverter non-idealities compensation', IEEE Trans. Ind. Appl., 2008, 44, (5), pp. 1587-1596

\section{Appendix}

See Tables 2 and 3

Table 2 Motor parameters and name plate data

\begin{tabular}{lcc}
\hline Parameter & Name & Value \\
\hline$R_{s}$ & stator resistance & $1.53 \Omega$ \\
$L_{d}$ & $d$-axis inductance & $16.07 \mathrm{mH}$ \\
$L_{q}$ & -axis inductance & $15.81 \mathrm{mH}$ \\
$\lambda_{\mathrm{mg}}$ & PM flux linkage & $0.165 \mathrm{Vs}$ \\
$p$ & pole pairs & 4 \\
$U_{\mathrm{dc}}$ & DC-bus voltage & $563 \mathrm{~V}$ \\
$U_{n}$ & nominal voltage & $254 \mathrm{~V}$ \\
$I_{n}$ & nominal current & $3.7 \mathrm{~A}$ \\
$I_{0}$ & $4.7 \mathrm{~A}$ \\
$\omega_{n}$ & zero-speed maximum current & $314 \mathrm{rad} / \mathrm{s}$ \\
$\lambda_{n}$ & nominal speed & $0.2021 \mathrm{Vs}$ \\
$\tau_{n}$ & nominal flux & $3.7 \mathrm{Nm}$ \\
$J_{m}$ & nominal torque & $1.6 \times 10^{-3} \mathrm{~kg} \mathrm{~m}$ \\
$B_{m}$ & moment of inertia & $7.48 \times 10^{-4} \mathrm{Nms} / \mathrm{rad}$ \\
\hline
\end{tabular}

Table 3 Flux estimator parameters

\begin{tabular}{lcc}
\hline Parameter & Name & Value \\
\hline$\omega_{0}$ & natural frequency & $125.7 \mathrm{rad} / \mathrm{s}$ \\
$\xi$ & damping factor & 0.707 \\
$K_{p}$ & Pl proportional gain & 0.09 \\
$K_{i}$ & Pl integral gain & $2.58 \mathrm{~s}$ \\
\hline
\end{tabular}

